\title{
Echo signal from rough planar interfaces influence of roughness, angle, range and transducer type
}

Wilhjelm, Jens E.; Pedersen, P.C.; Jacobsen, S.M.; Martinsen, Kjeld

Published in:

I E E E International Ultrasonics Symposium. Proceedings

Link to article, DOI:

10.1109/ULTSYM.1998.765309

Publication date:

1998

Document Version

Publisher's PDF, also known as Version of record

Link back to DTU Orbit

Citation (APA):

Wilhjelm, J. E., Pedersen, P. C., Jacobsen, S. M., \& Martinsen, K. (1998). Echo signal from rough planar interfaces influence of roughness, angle, range and transducer type. I E E E International Ultrasonics Symposium. Proceedings, 2, 1839 - 1842. https://doi.org/10.1109/ULTSYM.1998.765309

\section{General rights}

Copyright and moral rights for the publications made accessible in the public portal are retained by the authors and/or other copyright owners and it is a condition of accessing publications that users recognise and abide by the legal requirements associated with these rights.

- Users may download and print one copy of any publication from the public portal for the purpose of private study or research.

- You may not further distribute the material or use it for any profit-making activity or commercial gain

- You may freely distribute the URL identifying the publication in the public portal 


\title{
Echo Signal from Rough Planar Interfaces - Influence of Roughness, Angle, Range and Transducer Type
}

\author{
J. E. WILHJELM ${ }^{1}$, P. C. PEDERSEN ${ }^{2}$, S.-M. JACOBSEN ${ }^{1}$ \& K. MARTINSEN ${ }^{1}$ \\ Center for Arteriosclerosis Detection with Ultrasound (CADUS) \\ E-mail: wilhjelm@it.dtu.dk. Homepage: http://www.it.dtu.dk/ wilhjelm/cadus.html \\ ${ }^{1}$ Dept. of Information Tech., Tech. Univ. of Denmark, Bldg. 344, DK-2800 Lyngby, Denmark. \\ ${ }^{2}$ Elec. \& Comp. Eng. Dept., Worcester Polytechnic Institute, Worcester, MA 01609, USA.
}

\begin{abstract}
The received electrical signal from a pulse-echo system insonifying a planar acoustical interface was measured for varying degrees of rms roughness $(0-0.16$ $\mathrm{mm}$ ), angle of incidence (typically $+1-7^{\circ}$ ) and range to the transducer. A planar and a focused $5 \mathrm{MHz}$ transducer was used.

When insonifying a smooth interface, the normalized spectrum of the received signals for a planar transducer exhibits an increasing number of nulls with increased angle of insonification, as predicted from numerical modeling while the dependence on insonification angle for the focused transducer was smaller and the null pattern was much less distinct.

For the planar transducer and for the focused transducer with the interface located at the geometrical point of focus, the energy of the received signal as a function of incident angle was approximately Gaussian with maximum at $0^{\circ}$. For the smooth interface, the $-3 d B$ width was $\sim 0.5^{\circ}$ and $-3^{\circ}$ for the planar and the focused transducer, respectively.
\end{abstract}

\section{Introduction}

Tissue characterization has been an active area of research for several decades. The progress has, however, been limited by the fact that the 1D electrical signal from the receiving transducer is the result of not only the 3D distribution of scatterers, the geometry and acoustic properties of interfaces and scatterers, but also to a large extent the field pattern and frequency response of the given transducer and constructive/destructive interference in the backscattered field at the surface of the recejving transducer.

As an example, the received electrical signal from a pulse-echo system insonifying a planar acoustical interface depends mainly on surface characteristics, orientation of the interface and on transducer geometry. When the surface characteristics is limited to smooth, the given measurement configuration can be simulated with computer model $s^{[4]}$ even when the interface is non-planar ${ }^{[2]}$. When a rough planar surface is considered, the modeling becomes stochastic in nature ${ }^{[1]}$, and computer modeling tools are not yet available to readily predict the received electrical signal.
To attain an understanding of the received signal from a pulse-echo system as a function of transducer geometry and the roughness properties of the reflector, received signals were acquired for planar interfaces with varying degrees of $\mathrm{rms}$ roughness, angle of incidence, and range to the transducer, as depicted in Figure 1.

The information derived from such measurements might have potential applications in NDE and - on a larger time horizon - characterization of pathological changes in tissue interfaces, e.g., artery walls. This quantitative information can also serve as a reference when evaluating future simulation approaches to this problem.

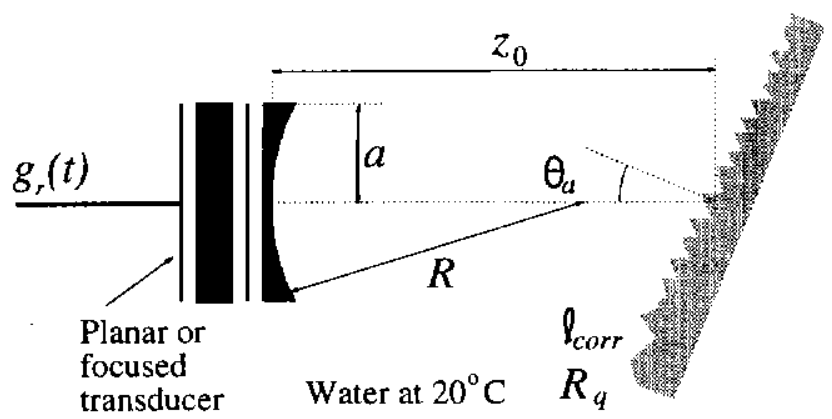

Figure 1 Side view of the basic measurement set-up. A rough planar interface with $r m s$ roughness $R_{q}$ and correlation length $\ell_{c a r r}$ is rotated the angle $\theta_{a}$ at a distance $z_{0}$ from a planar or focused transducer (radius of curvature, $R$ ) with radius $a$.

\section{Reflectors and Ultrasound System}

Four rough and one smooth reflector were made, using four types of sandpaper with different degrees of roughness as molds, and casting with a two component, liquid urethane casting elastomer (Biresin ${ }^{\circledR}$ (type U1402, Sika Chemie GMBH, Stuttgart, Germany)). This elastomer has acoustic properties very close to those of human tissues (speed of sound, $c=1450 \mathrm{~m} / \mathrm{s}$; density, $\rho=1.06 \cdot 10^{3}$ $\mathrm{kg} / \mathrm{m}^{3} @ 20^{\circ} \mathrm{C}$ ). The molding took place in vacuum to ensure that the phantom was an exact inverse copy of the sandpaper surface. It was verified that the reflectors contained no voids or particles, so that a single acoustic interface between water and elastomer was obtained 
during measurements. The $r m s$ roughness and correlation length was calculated from the measurement of several profiles directly on the sandpaper with a laser profilometer (type OTM3-10/144, UBM Messtechnik, Ettlingen, Germany) working in triangulation mode.

The electrical part of the measurement system is depicted in Figure 2. The ultrasound system consisted of a pulser/receiver connected a submersible transducer. The amplified signal from the pulser/receiver was bandpass filtered to limit noise outside the passband of the transducer and digitized with a digital storage oscilloscope, DSO. The DSO was in turn connected via a GPIB interface to a Windows $\mathrm{NT}^{\mathrm{TM}}$ based workstation. By use of an RS232 interface, a 3D translation system was connected to this workstation as well.

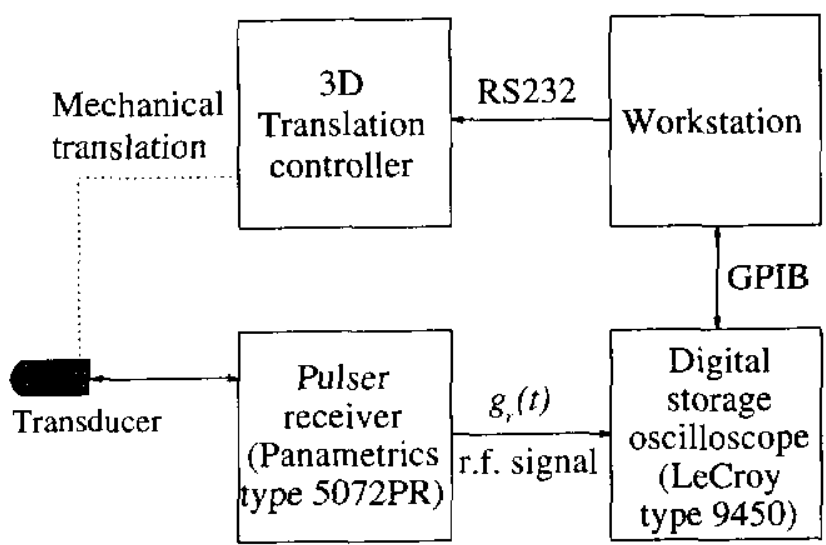

Figure 2 Block diagram of the electrical part of the measurement system. A workstation is controlling the transducer position and reading the digitized signals from the digital storage oscilloscope.

The experimental set-up is illustrated in Figure 3. The transducer was mounted to the $3 \mathrm{D}$ translation system and adjusted such that its acoustic axis was parallel to the $x_{t}$-axis. A dual-angle rotation holder (Gimbal Jig) was fixed to the scanning tank. It consisted of two frames that could be rotated independently of each other with the two axes of rotation normal to each other. The rough reflector was placed in the holder such that the two axes of rotation were in the plane of the surface. Measurements took place in degassed demineralized water at $20^{\circ} \mathrm{C}$.

\section{Measurement Procedure}

The transducer was adjusted so that its acoustic axis was parallel to the $x_{t}$-axis shown in Figure 3 . In order to identify $0^{\circ}$ for the azimuth and elevation angles, the transducer was moved in front of the inner frame, and the delay between the emitted pulse and the reflected pulse from the part of the inner frame that faced the transducer was recorded. These delays were measured at four corners, and the angles adjusted until all four delay values

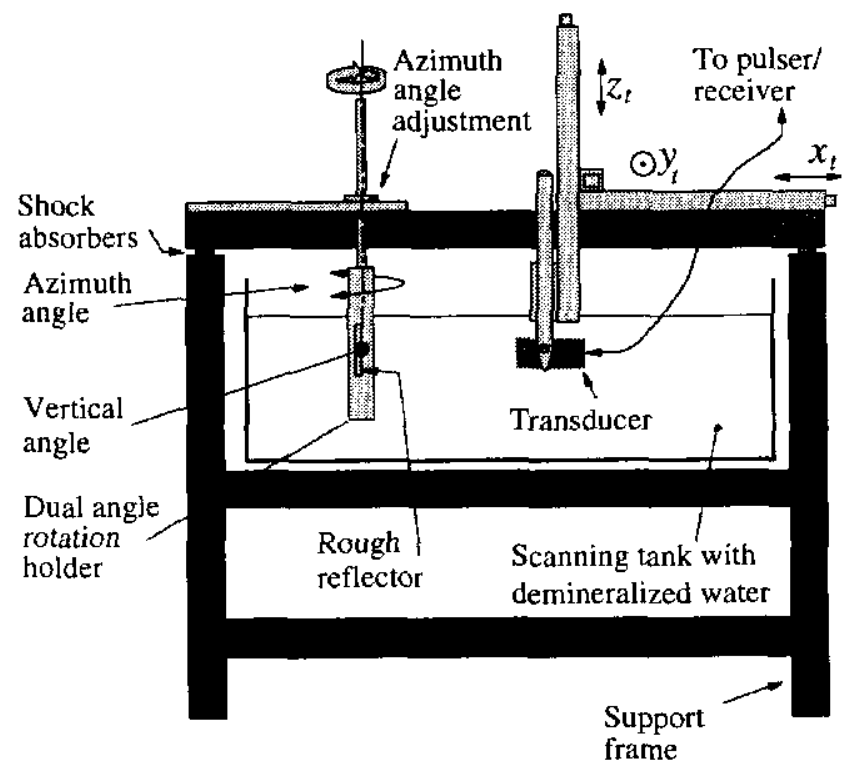

Figure 3 The experimental system showing reflector in dual-angle rotation holder and transducer, both submerged in demineralized degassed water.

were equal. The vertical angle was then locked. The smooth reflector was next inserted and the control procedure was repeated on the smooth surface.

The angular span, $\pm \theta_{a, \max }$ was set according to the specific transducer used. The increment, $\Delta \theta_{u}$, was variable over the angular recording range, so that the fine structure of the changes in the received signal as a function of angle could be recorded. At measurement start, the azimuth angle was set to $-\theta_{a, m a x}$ and increased to $\theta_{a, m a x}$ in increments of $\Delta \theta_{a}\left(\theta_{a}\right)$, by turning a micrometer screw that translated an arm mounted to the axis of azimuthal rotation.

Because air can be trapped at the surface of the rough reflectors these were degassed in a water bath in vacuum prior to use (by using simple manual manipulation under water, it was only possible to successfully remove air from the surface of the smooth reflector).

For each transducer, the rough reflector surfaces were divided into $M$ equally spaced insonified regions, and one echo signal was recorded from the center of each region. For each angle and range, it was thus possible to record several received signals and from these obtain statistically stable estimates of the signal parameters.

\section{Signal Processing}

The received signal for the $m$ 'th surface cell, at range $z_{0}$ from the reflector, rotated the angle $\theta_{a}$, is denoted $g_{r}\left(t ; m, \theta_{t p} z_{w} R_{q}\right)$, where $R_{4}$ is the rms roughness of the interface and $m \in[1 ; M]$. The normalized mean amplitude spectrum is 


$$
\begin{aligned}
& G_{\text {Norm }}\left(f ; \theta_{a}, z_{0}, R_{q}\right) \\
& =\frac{\frac{1}{M} \sum_{m=1}^{M}\left|\mathrm{FT}\left\{g_{r}\left(t ; m, \theta_{a}, z_{0}, R_{q}\right)\right\}\right|}{\left|\mathrm{FT}\left\{g_{r}\left(t ; 1,0, z_{0}, 0\right)\right\}\right|}
\end{aligned}
$$

where $g_{r}\left(t ; 1,0, z_{0}, 0\right)$ is a single received signal from a smooth reflector at normal incidence and FT $\{\cdot\}$ denotes Fourier Transform. The mean energy of the received signals was calculated from

$E\left(\theta_{a}, z_{0}, R_{q}\right)=\frac{1}{M} \sum_{m=1}^{M} \int g_{r}^{2}\left(t ; m, \theta_{a}, z_{0}, R_{q}\right) d t$

where the integration covers the entire echo signal from the interface.

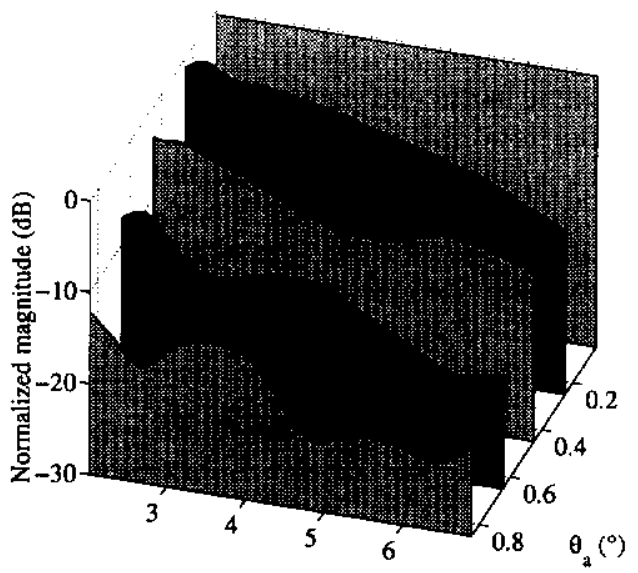

(a)

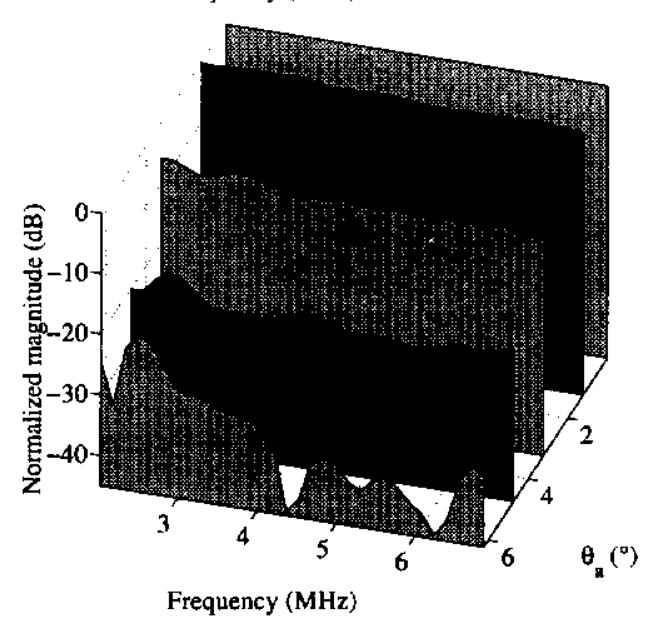

(c)

\section{5 . Results}

Results are provided for a planar and a focused $5 \mathrm{MHz}$ transducer, both with radius of the active element, $a=$ $12.7 \mathrm{~mm}$ (Panametrics Inc., type V307). Radius of curvature for the focused transducer was $R=118 \mathrm{~mm}$. The interface was located at range $z_{0}=50 \mathrm{~mm}$ from the flat transducer and at range $z_{0}=R$ from the focused transducer. The wavelength at $5 \mathrm{MHz}$ in $20^{\circ} \mathrm{C}$ pure water is approximately $300 \mu \mathrm{m}$.

The normalized amplitude spectra for the planar and corresponding focused transducer are shown in Figure 4 for a smooth and a rough reflector. The normalized energy of the echo signal as a function of angle for the planar and the focused transducer is shown in Figure 5 for several degrees of roughness. All curves are normalized to the same maximum.

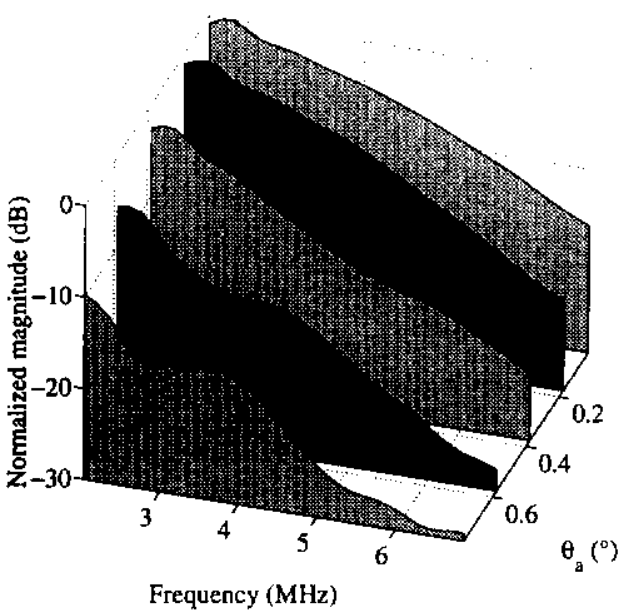

(b)

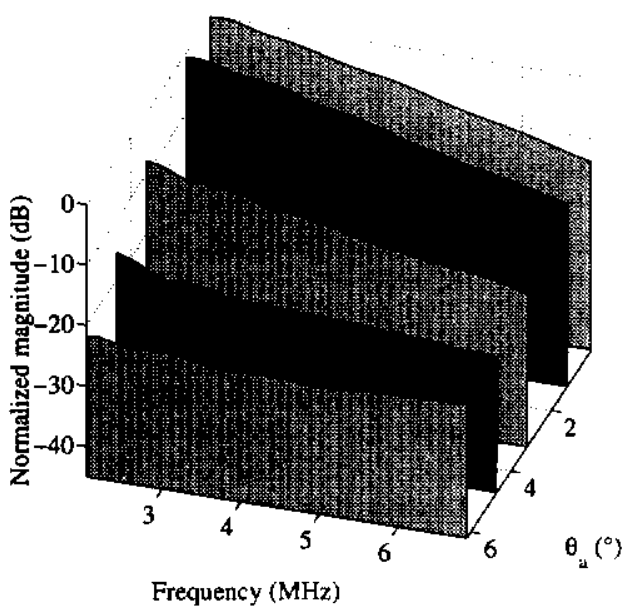

(d)

Figure 4 Normalized spectra, $G_{\text {Norm }}\left(f ; \theta_{a}, z_{0}, R_{q}\right)$, in $\mathrm{dB}$ for a planar (a) and (b), and a focused (c) and (d) transducer ( $z_{0}$ $=R$ ). (a) and (c) are for a smooth interface $(M=1)$, while (b) and (d) are for an interface with $R_{q}=32 \mu \mathrm{m}=\lambda / 10 @$ $5 \mathrm{MHz}$. 


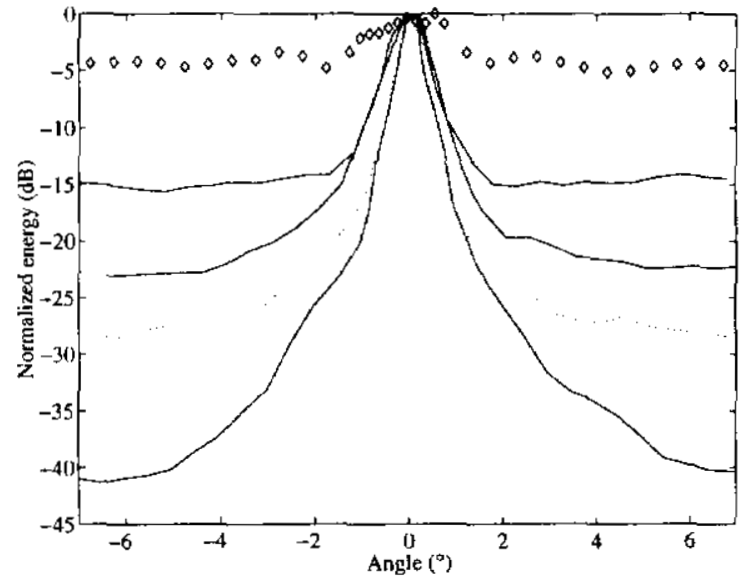

(a)

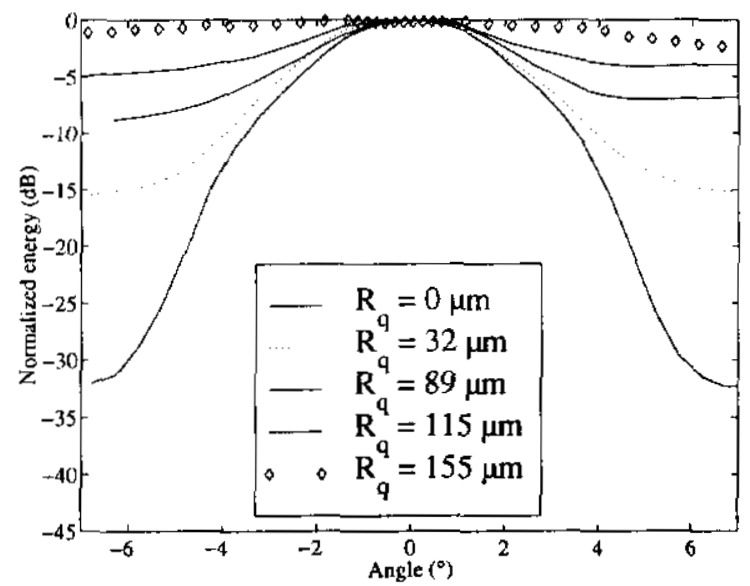

Figure 5 Energy of received signal, $E\left(\theta_{a}, z_{1}, R_{q}\right)$, from planar interfaces with various degrees of roughness for the planar (a) and the focused (b) transducer $\left(z_{1}=R\right) . R_{q}$ for both sets of curves is given in the legend box in (b).

\section{Discussion}

Normalized spectrum, planar transducer: For the smooth interface, the normalized spectrum has a negative slope and exhibits an increasing number of nulls with increased angle, as predicted from numerical modeling ${ }^{[3]}$. There are some minor deviations which are probably due to the transducer's non-ideal behavior. Increasing the rms roughness made the null locations less distinct as seen in Figure $4 \mathrm{~b}$. Nevertheless, the overall picture remains the same, indicating that at $R_{q}=\lambda / 10$, the echo signal is still governed mainly by specular reflection. These observations suggest that the reduction in received signal energy with increased incident angle for a smooth surface is attributable to coherent phase cancellation over the surface of the transducer. As the $r m s$ roughness of the reflector becomes larger, the reflected pressure field becomes more incoherent, and hence the phase cancellation with increased incident angle is reduced.

Normalized spectrum, focused transducer: For the smooth interface, the angle-dependence was reduced compared to the planar transducer and the null pattern was much less distinct (Figure $4 \mathrm{c}$ ). At $\theta_{a} \cong 6^{\circ}$, strong phase cancellation is seen together with an overall attenuation of $\sim 30 \mathrm{~dB}$. For the rough interface at lower angles, the specular reflection is slowly substituted with weaker scattering, as the frequency increases yielding the negative slope of the spectra in Figure 4d. At $\theta_{a} \cong 6^{\circ}$, a comparison of Figure $4 \mathrm{c}$ with $\mathrm{d}$, shows how the scattering component maintains a nearly constant spectrum.

Energy of the received signal: The energy as a function of angle has a nearly Gaussian shape with maximum at $0^{\circ}$. For the smooth interface, the $-3 \mathrm{~dB}$ width was $\sim 0.5^{\circ}$ for the planar transducer and $\sim 3^{\circ}$ for the focused transducer $\left(z_{0}=R\right)$. The $-3 \mathrm{~dB}$ width increases exponentially with $r m s$ roughness so that when $R_{q} \cong 155 \mu \mathrm{m} \cong \lambda_{q} / 2$, the energy is nearly constant inside the angular interval considered. From results not shown here, it was found that while $E\left(\theta_{a}, z_{0}, R_{q}\right)$ is rather constant with $z_{0}$ for a planar transducer, $E\left(\theta_{a}, z_{0}, R_{q}\right)$ goes through some dramatical changes when $z_{1}<R$ for a focused transducer.

\section{Conclusions}

Received echo signals have been recorded from planar interfaces with varying degrees of roughness at different insonification angles and ranges. The results show that the echo signal from planar transducers are more sensitive to changes in insonification angle (around $0^{\circ}$ ) than focused transducers with the reflecting interface located at the focal point. As shown quantitatively, the angledependence decreases when the rms roughness of the reflecting interface increases.

\section{Acknowledgements}

The help from J. Keiding and A. Albertsen, Leo Pharmaceutical Products, Ballenup, Denmark for obtaining the profilometer results is gratefully acknowledged. CADUS is supported by the Danish Technical and Medical Research Councils.

\section{References}

[1] Dean BJ, Pedersen PC: Angular Spectrum Based Formulation of Rough Surface Scattering With Applications to Surface Characterization. Proc. of the 1996 IEEE Int. Ultrason. Symp., Nov. 3 - 6, pp. 693 - 696. 1996.

[2] Jespersen SK, Pedersen PC and Wilhjelm JE: The Diffraction Response Interpolation Method. Accep. for publ. in IEEE Trans. Ultrason., Ferroelect. and Freq. Contr. November, 1998.

[3] Orofino DP, Pedersen PC: Angle-Dependent Spectral Distortion for an Infinite Planar Fluid-Fluid Interface. J. Acoust. Soc. Am., vol. 92, no. 5, pp. 2883-2899. 1992.

[4] Pedersen PC, Orofino DP: Modeling of Received Ultrasound Signals from Finite Planar Targets. IEEE Trans. Ultrason., Ferroelect. and Freq. Contr. vol. 43, no. 2, pp. 303-311. 1996. 